\title{
Effect of dietary fatty acid intake on prospective weight change in the Heidelberg cohort of the European Prospective Investigation into Cancer and Nutrition
}

\author{
Katharina Nimptsch 1,2, Gabriele Berg-Beckhoff ${ }^{3}$ and Jakob Linseisen 1,4,* \\ 'Division of Cancer Epidemiology, German Cancer Research Center, Im Nevenheimer Feld 280, Heidelberg, \\ Germany: ${ }^{2}$ Department of Nutrition, Harvard School of Public Health, 665 Huntington Avenue, Boston, MA, \\ USA: ${ }^{3}$ Department of Epidemiology and International Public Health, Faculty of Public Health, University of \\ Bielefeld, Bielefeld, Germany: ${ }^{4}$ Helmholtz Zentrum München (HMGU), Institute of Epidemiology, Ingolstädter \\ Landstr. 1, D-85764 Neuherberg, Germany
}

Submitted 16 February 2009: Accepted 26 November 2009: First published online 15 January 2010

\begin{abstract}
Objective: To evaluate the association between fatty acid ( $\alpha$-linolenic acid (ALA), EPA, DHA, palmitic, stearic, oleic, linoleic and arachidonic acids) intake and prospective weight change in the Heidelberg cohort of the European Prospective Investigation into Cancer and Nutrition.

Design: Prospective cohort study with mean follow-up time of 6.5 years. In a total of 9182 men and 10867 women aged 35 to 64 years, from body weight measurement at recruitment and calibrated body weight during follow-up, weight change was expressed as mean annual weight change relative to baseline weight (\%/year) and categorised into four groups (weight loss, $<-2.5 \% / 5$ years; stable weight, between -2.5 and $+2.5 \% / 5$ years; small weight gain, $\geq 2.5$ to $<7.5 \% / 5$ years; large weight gain, $\geq 7 \cdot 5 \% / 5$ years). Energy-adjusted dietary fatty acid intake data were estimated from the FFQ completed at baseline. Multivariate linear regression models as well as multinomial logistic regression analyses (carbohydrate replacement models) were conducted.

Results: Stearic acid intake was linearly associated with weight gain $(P<0 \cdot 01)$ in men and women. Linear associations also existed for ALA and arachidonic acid intake, significantly so in women. In multinomial models, women in the highest tertile of ALA and stearic acid intake showed increased OR (95\% CI) for small weight gain $(1 \cdot 16(0 \cdot 94,1 \cdot 88)$ and $1 \cdot 24(1 \cdot 08,1 \cdot 43)$, respectively), and large weight gain $(1.39(1.03,1.88)$ and $1.56(1.27,1.90)$, respectively), whereas OR were non-significantly increased in men. Dietary intake of ALA was inversely associated with large $(0 \cdot 80(0 \cdot 65,0 \cdot 99))$ weight gain in women only.

Conclusions: These results suggest differential effects of single dietary fatty acids on prospective weight gain in adults.
\end{abstract}

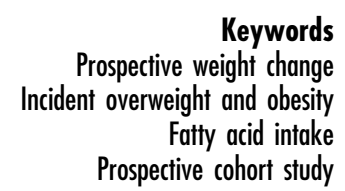

The prevalence of overweight and obesity has increased remarkably during the past 20 years in Germany and worldwide ${ }^{(1,2)}$. Two-thirds of all obese adults have developed obesity during adulthood ${ }^{(3)}$. Since the proportion of overweight people is lowest at the age of 18-19 years ${ }^{(4)}$, maintenance of the body weight after adolescent growth should be the primary aim of obesity prevention in adults ${ }^{(5)}$.

In principle, weight change occurs when there is an imbalance between energy intake and energy expenditure over a longer time period. Psychosocial or genetic factors favouring excess energy intake and reduced energy expenditure can contribute to a positive energy balance eventually leading to weight gain ${ }^{(6)}$. Established determinants of weight change are alcohol consumption, smoking habits, sociodemographic factors, physical activity, mental stress, voluntary weight loss or dieting behaviour $^{(7)}$. The role of diet in weight change is complex and still many aspects remain to be elucidated. Fat is the most energy-dense macronutrient providing $38 \mathrm{~kJ} / \mathrm{g}$ (9 kcal/g) and high-fat foods are characterised by enhanced flavour and palatability, while having a less satiating effect per kilojoule than low-fat foods rich in protein or complex carbohydrates; thus, a diet with a high proportion of fat is prone to lead to overconsumption of energy $^{(8)}$. Although long-chain fatty acids differ only slightly with respect to the energy content, they can vary in their effect on energy balance. Both energy expenditure and satiety, which influence the magnitude of excess 
energy intake, have been shown to be affected by the diet's fatty acid composition ${ }^{(9)}$. The mechanisms by which the fatty acids may impact on energy expenditure include their effect on postprandial thermogenesis ${ }^{(10)}$, fat oxidation rate ${ }^{(11,12)}$ and the sympathetic activity ${ }^{(13)}$. So far, observational studies in human subjects have investigated whether different types of fatty acids (i.e. PUFA $v$. MUFA $v$. SFA) differ in their effect on body weight ${ }^{(8,14)}$ or weight change $^{(15)}$. However, data from experimental studies suggest that the effects of individual fatty acids are not necessarily uniform ${ }^{(16)}$. Therefore, in the present study, the hypothesis that individual fatty acids have differential effects on long-term weight change was tested within a prospective cohort study, the Heidelberg cohort of the European Prospective Investigation into Cancer and Nutrition (EPIC-Heidelberg). On the basis of the results of such observational studies, properly designed metabolic/ intervention studies should follow.

\section{Participants and methods}

\section{Study population and data collection}

The EPIC-Heidelberg comprises 11928 men (aged 40-64 years) and 13612 women (aged 35-64 years), recruited between 1994 and 1998 (66626 volunteers were contacted, overall participation rate was $38 \cdot 3 \%$ ). Habitual dietary intake during the 12 months preceding the recruitment was assessed by a validated self-administered 158-item semi-quantitative FFQ including additional questions with regard to the type of fat for cooking and fat content of milk and milk products ${ }^{(17)}$. Food coding and calculation of the individual intake of fatty acids and other nutrients were carried out using the German Food Code (version II.3) ${ }^{(18)}$. Information on lifestyle factors such as educational attainment, smoking habits and physical activity was assessed by self-administered questionnaires and a personal computer-guided interview at baseline. During the participant's visit in the study centre, body weight was measured on digital scales (Soehnle, type $7720 / 23$, Murrhardt, Germany), and height was measured using a flexible anthropometer by educated interviewers. In the follow-up questionnaires, which are mailed to the participants at regular intervals, participants were asked to report their current body weight. The participation rates during the follow-ups were above $90 \%$ at all times. The study was approved by the local ethics committee and all participants gave their informed consent.

\section{Calibration of self-reported body weight at follow-up}

To correct for biases in the self-reported weight, to data from the three follow-up rounds, a calibration method was applied, as described by Spencer et al. ${ }^{(19)}$, using data from the subsample of volunteers who participated in the
$24 \mathrm{~h}$ dietary recalls conducted by trained interviewers at baseline ( $n$ 2121). During the recalls, participants gave self-reported information on their body weight at baseline. In a simple regression analysis, measured weight was modelled as a function of age and self-reported weight, stratified by sex and standard BMI categories $\left(<25,25-29 \cdot 9, \geq 30 \mathrm{~kg} / \mathrm{m}^{2}\right)$. The obtained equations were used to calibrate the self-reported weight data of the follow-up, assuming a consistency in bias in the selfreported values at baseline and follow-up. Comparison of reported and calibrated follow-up weight revealed that, on average, men tended to underestimate their follow-up weight less than women: the mean difference between calibrated and reported weight was 0.72 (SD 0.59), 0.67 $(\mathrm{SD} 0 \cdot 60), 0 \cdot 65 \mathrm{~kg}(\mathrm{SD} 0 \cdot 60)$ in men and $1 \cdot 07$ (SD 0.70), 1.03 (sD 0.70$), 0.98 \mathrm{~kg}(\mathrm{SD} 0.68)$ in women, in the first (1998-2000), second (2001-2004) and third (2004-2007) follow-up, respectively.

\section{Statistical analyses}

Follow-up time was defined as the time between baseline interview and last follow-up contact. Weight change was calculated as the difference between calibrated selfreported body weight at the last completed follow-up and measured body weight at baseline. For the analysis, annual weight change was expressed as a percentage of baseline body weight to take into account that absolute weight change is strongly dependent on baseline weight. Four categories of weight change in the percentage of baseline weight were defined as follows: stable body weight (weight change between -2.5 and $+2.5 \% / 5$ years); weight loss (weight change $<-2.5 \% / 5$ years); small weight gain (weight change of $\geq 2.5$ to $<7.5 \% / 5$ years) and large weight gain (weight change $\geq 7.5 \% / 5$ years). Change in smoking status between baseline and last follow-up was accounted for as follows: smokers at baseline and last follow-up ('habitual smokers'), nonsmokers at both assessment periods ('habitual nonsmokers'), smokers at baseline, reporting non-smoking at follow-up ('quitters') and non-smokers at baseline and reporting smoking at follow-up ('new smokers'). Physical activity was classified into four categories (inactive, moderately inactive, moderately active and active), combining both occupational and recreational activities ${ }^{(20)}$.

All nutrient values were energy-adjusted by the residual method as described by Willett and Stampfer ${ }^{(21)}$. The association between fatty acid intake (palmitic, stearic, oleic, linoleic, arachidonic, $\alpha$-linolenic acid (ALA), EPA and DHA) and prospective weight change was investigated using linear and multinomial logistic regression models. In multiple linear regression analyses, a 5-year weight change was modelled as a continuous dependent variable. Nutrient intakes were entered as continuous independent variables into the model. Participants who lost more than $2.5 \%$ of their baseline weight (weight losers) during 5 years of follow-up were excluded from 
this analysis to focus on weight gain. In a subanalysis, only participants with normal weight (BMI between $18 \cdot 5$ and $25 \mathrm{~kg} / \mathrm{m}^{2}$ ) at baseline were included.

For the multinomial logistic regression, generalised logits were modelled for the three weight change categories $v$. stable weight. OR and 95\% CI were calculated by tertiles of fatty acid intake with the lowest tertile being the reference category. Risk estimates for small and large gain are given in the Results section (results for weight loss are not shown). A test for trend across increasing tertiles of fatty acid intake was performed by entering median intake values of the fatty acid tertiles as a continuous variable in the multivariate models. In the multinomial logistic regression analysis, weight change is treated as a categorical outcome variable. Participants with stable weight are considered the outcome reference corresponding to non-diseased participants in classical binary logistic regression models. The OR can be interpreted as the relative risk to fall into a certain weight change category according to tertiles of dietary fatty acid intake with the lowest tertile being the reference.

Isoenergetic nutrient residual models were created by entering the energy-adjusted intakes of all fatty acids of interest (as continuous variable or tertile categories for the linear and multinomial logistic regression, respectively) simultaneously together with the intake of other SFA, other MUFA, other PUFA, protein and alcohol, as well as total energy intake. The coefficients derived from these models represent the effect of substituting energy from carbohydrates by energy from a specific fatty acid ${ }^{(22)}$. As intake of EPA and DHA was highly correlated $(r>0 \cdot 97)$, their intake was entered into the model as the sum of both. All models were adjusted for weight and height at baseline, age, physical activity, education, change in smoking status and length of follow-up. In women, multivariate models were additionally adjusted for menopausal status at baseline (premenopausal, perimenopausal, postmenopausal, surgical menopausal and missing information). The analyses were repeated with a model representing the effect of substituting energy from MUFA by energy from specific fatty acids (adjusted for intakes of other PUFA, other SFA, carbohydrates, protein, alcohol and total energy). In contrast to the carbohydrate replacement model in the MUFA replacement model, the proportion of energy from fat is held constant.

In a final approach, incident overweight and obesity, respectively, were treated as outcomes in a binary logistic regression. Participants were defined as incident overweight or obese if they had a BMI $<25$ or $<30 \mathrm{~kg} / \mathrm{m}^{2}$, respectively, at baseline and a BMI of $\geq 25$ or $\geq 30 \mathrm{~kg} / \mathrm{m}^{2}$, respectively, at last follow-up. For this analysis, participants who were overweight or obese, respectively, at baseline were excluded. Adjustment was identical to the multinomial logistic regression analyses.

All statistical analyses were performed by the SAS statistical software package version 9.1 (SAS Institute, Cary, NC, USA, 2002). Analyses were performed separately for men and women. Statistical tests were two-sided, and significance level was set at $P<0 \cdot 05$.

\section{Results}

Participants who were lost to follow-up or did not report their body weight in at least one of the follow-up rounds ( $n$ 176) and participants who were predisposed to weight change because of prevalent or incident cancer, myocardial infarction, stroke or inflammatory bowel disease ( $n$ 4904) were excluded, as well as the top and bottom percentile of weight change (in percentage of baseline weight, $n$ 411). Finally, 20049 participants (9182 men and 10867 women) were included in the analysis.

Characteristics of the study population including dietary intake of the fatty acids of interest are presented in Table 1. Male participants were older and had a higher BMI than female participants. The proportion of normal weight participants at baseline was $55 \cdot 3 \%$ in women, but only $32.0 \%$ in men. On average, women gained, in a 5-year interval of follow-up, $1 \cdot 6 \%$ of baseline weight compared with $0 \cdot 8 \%$ in men. The proportion of participants with a baseline BMI $<25 \mathrm{~kg} / \mathrm{m}^{2}$ who became overweight during follow-up (incident overweight) was $19 \cdot 9 \%$ and $12 \cdot 4 \%$ in men and women, respectively. Around $5 \%$ of all men and women, who had a BMI $<30 \mathrm{~kg} / \mathrm{m}^{2}$ at baseline, were obese at their last follow-up (incident obese). Mean follow-up time was 6.5 years for both men and women.

Table 2 shows the distribution of the weight change categories. Stable weight participants form the largest group with a proportion of $50 \cdot 7 \%$ and $42.7 \%$ in men and women, respectively. About $18 \%$ of male and female participants had a 5 -year weight loss of more than $2 \cdot 5 \%$. More women (39.8\%) than men $(31 \cdot 1 \%)$ were characterised by a 5-year weight gain. The proportion of men and women who gained weight during follow-up decreased with increasing baseline age. Age at baseline and the percentage of weight change were significantly inversely correlated in men and women $(P<0 \cdot 0001)$.

The results of the linear regression analyses modelling the influence of fatty acid intake on prospective weight gain in men and women are presented in Table 3. Stearic acid intake was significantly positively associated with weight gain, yielding a weight gain $>3 \%$ in men and women per $10 \mathrm{~g}$ increment ( $>4 \%$ in normal weight participants at baseline). For a better illustration, a participant with a baseline body weight of $100 \mathrm{~kg}$ would gain an additional $3 \mathrm{~kg}$ over 5 years by increasing stearic acid intake by $10 \mathrm{~g} / \mathrm{d}$ (at the expense of an equal proportion of energy from carbohydrates); a participant with $70 \mathrm{~kg}$ would increase body weight by approximately $2 \mathrm{~kg}$. In addition, the dietary intake of the $n-6$ PUFA linoleic acid (only significant in women) and arachidonic acid was significantly associated with prospective weight gain, more pronounced in normal weight participants at 
Table 1 Characteristics of male and female study participants (EPIC-Heidelberg)

\begin{tabular}{|c|c|c|c|c|c|}
\hline & \multicolumn{2}{|c|}{ Men } & \multicolumn{2}{|c|}{ Women } & \multirow[b]{2}{*}{$P$ value } \\
\hline & Mean or \% & SD & Mean or \% & SD & \\
\hline$n$ & \multicolumn{2}{|c|}{9182} & \multicolumn{2}{|c|}{10867} & \\
\hline Age at baseline (years) & $51 \cdot 4$ & $7 \cdot 1$ & $48 \cdot 4$ & $8 \cdot 5$ & $<0.001^{*}$ \\
\hline Baseline body weight $(\mathrm{kg})$ & $83 \cdot 3$ & $12 \cdot 0$ & $67 \cdot 7$ & $12 \cdot 2$ & $<0.001^{*}$ \\
\hline Baseline BMI $\left(\mathrm{kg} / \mathrm{m}^{2}\right)$ & $26 \cdot 8$ & $3 \cdot 6$ & $25 \cdot 2$ & $4 \cdot \overline{5}$ & \\
\hline Underweight (BMI $\left.<18.5 \mathrm{~kg} / \mathrm{m}^{2}, \%\right)$ & $0 \cdot 1$ & & $1 \cdot 4$ & & \\
\hline Normal weight $\left(18.5 \leq \mathrm{BMI}<25 \mathrm{~kg} / \mathrm{m}^{2}, \%\right)$ & $32 \cdot 0$ & & $55 \cdot 3$ & & \\
\hline Overweight $\left(25 \leq \mathrm{BMl}<30 \mathrm{~kg} / \mathrm{m}^{2}, \%\right)$ & $51 \cdot 1$ & & $29 \cdot 3$ & & \\
\hline Obese $\left(\mathrm{BMI} \geq 30 \mathrm{~kg} / \mathrm{m}^{2}, \%\right)$ & $16 \cdot 8$ & & $14 \cdot 0$ & & $<0.001+$ \\
\hline Follow-up body weightł $(\mathrm{kg})$ & $84 \cdot 1$ & $12 \cdot 1$ & $68 \cdot 9$ & $12 \cdot 5$ & $<0.001^{*}$ \\
\hline Follow-up BMI $\left(\mathrm{kg} / \mathrm{m}^{2}\right)$ & $27 \cdot 1$ & $3 \cdot 7$ & $25 \cdot 7$ & $4 \cdot 7$ & $<0 \cdot 001^{*}$ \\
\hline Five-year weight change (kg/5 years) & $0 \cdot 6$ & $3 \cdot 9$ & $1 \cdot 0$ & $3 \cdot 6$ & $<0 \cdot 001^{*}$ \\
\hline Five-year weight change (\%/5 years) & $0 \cdot 8$ & $4 \cdot 5$ & $1 \cdot 6$ & $5 \cdot 1$ & $<0.001^{*}$ \\
\hline Incident overweight (\%) & $19 \cdot 9$ & & $12 \cdot 4$ & & $<0.001 \dagger$ \\
\hline Incident obesity (\%) & $5 \cdot 0$ & & $4 \cdot 7$ & & $<0.001 \dagger$ \\
\hline Length of follow-up (years) & $6 \cdot 5$ & $2 \cdot 2$ & $6 \cdot 5$ & $2 \cdot 1$ & $<0 \cdot 83^{*}$ \\
\hline \multicolumn{6}{|l|}{ Nutrient intake } \\
\hline Total energy $(\mathrm{kcal} / \mathrm{d}) \S$ & 9204 & 3263 & 7237 & 2449 & $<0.001 \|$ \\
\hline Carbohydrates $(\mathrm{g} / \mathrm{d})$ & $236 \cdot 1$ & $91 \cdot 4$ & $193 \cdot 6$ & $72 \cdot 4$ & $<0.001 \|$ \\
\hline Protein $(\mathrm{g} / \mathrm{d})$ & 78.5 & $28 \cdot 3$ & $62 \cdot 4$ & $20 \cdot 9$ & $<0 \cdot 001 \|$ \\
\hline Alcohol (g/d) & $25 \cdot 8$ & $27 \cdot 2$ & $11 \cdot 0$ & $14 \cdot 2$ & $<0.001 \|$ \\
\hline Total fat $(\mathrm{g} / \mathrm{d})$ & $84 \cdot 6$ & $37 \cdot 7$ & $69 \cdot 8$ & $28 \cdot 7$ & $<0.001 \|$ \\
\hline (\% Energy) & $34 \cdot 4$ & $6 \cdot 0$ & $36 \cdot 1$ & $5 \cdot 6$ & $<0.001 \|$ \\
\hline SFA $(g / d)$ & $35 \cdot 1$ & $16 \cdot 8$ & $29 \cdot 6$ & $13 \cdot 3$ & \\
\hline (\% Energy) & $14 \cdot 2$ & $3 \cdot 0$ & $15 \cdot 2$ & $3 \cdot 0$ & $<0.001 \|$ \\
\hline Palmitic acid (g/d) & $17 \cdot 0$ & $7 \cdot 9$ & $14 \cdot 2$ & $6 \cdot 1$ & $<0.001 \|$ \\
\hline Stearic acid $(\mathrm{g} / \mathrm{d})$ & $7 \cdot 6$ & $4 \cdot 2$ & $6 \cdot 1$ & $3 \cdot 2$ & $<0.001 \|$ \\
\hline MUFA $(g / d)$ & $29 \cdot 9$ & $14 \cdot 0$ & $24 \cdot 1$ & $10 \cdot \overline{3}$ & \\
\hline (\% Energy) & $12 \cdot 1$ & $2 \cdot 4$ & $12 \cdot 4$ & $2 \cdot 2$ & $<0.001 \|$ \\
\hline Oleic acid $(\mathrm{g} / \mathrm{d})$ & $26 \cdot 2$ & $12 \cdot 4$ & $21 \cdot 0$ & $9 \cdot 1$ & $<0.001 \|$ \\
\hline PUFA $(g / d)$ & $14 \cdot 0$ & $6 \cdot 3$ & $11 \cdot 5$ & 4.9 & \\
\hline (\% Energy) & $5 \cdot 7$ & $1 \cdot 6$ & $6 \cdot 1$ & $1 \cdot 6$ & $<0.001 \|$ \\
\hline \multicolumn{6}{|l|}{$n-6$ PUFA } \\
\hline Linoleic acid (g/d) & $11 \cdot 9$ & $5 \cdot 5$ & $9 \cdot 9$ & $4 \cdot 3$ & $<0.001 \|$ \\
\hline Arachidonic acid (mg/d) & $201 \cdot 5$ & $105 \cdot 9$ & $143 \cdot 5$ & $81 \cdot 1$ & $<0.001 \|$ \\
\hline \multicolumn{6}{|l|}{$n$-3 PUFA } \\
\hline $\operatorname{ALA}(\mathrm{g} / \mathrm{d})$ & $1 \cdot 4$ & 0.6 & $1 \cdot 2$ & 0.5 & $<0.001 \|$ \\
\hline EPA $(\mathrm{mg} / \mathrm{d})$ & $102 \cdot 6$ & $128 \cdot 8$ & $69 \cdot 7$ & $106 \cdot 3$ & $<0.001 \|$ \\
\hline DHA (mg/d) & $201 \cdot 3$ & $185 \cdot 6$ & $146 \cdot 9$ & $152 \cdot 5$ & $<0.001 \|$ \\
\hline Physically active & $27 \cdot 0$ & & $24 \cdot 1$ & & $<0.001 \dagger$ \\
\hline With university degree & $38 \cdot 6$ & & $25 \cdot 3$ & & $<0.001 t$ \\
\hline Habitual non-smokers & $71 \cdot 4$ & & $75 \cdot 9$ & & \\
\hline Habitual smokers & $19 \cdot 3$ & & $16 \cdot 5$ & & $<0.001 t$ \\
\hline Premenopausal & & & $47 \cdot 8$ & & \\
\hline Perimenopausal & & & $11 \cdot 4$ & & \\
\hline Postmenopausal & & & $38 \cdot 8$ & & \\
\hline
\end{tabular}

EPIC-Heidelberg, Heidelberg cohort of the European Prospective Investigation into Cancer and Nutrition; ALA, $\alpha$-linolenic acid. ${ }^{*} t$-test. Data are presented as mean and standard deviation, or percentage. tChi-square test.

‡Calibrated.

$\S 1 \mathrm{kcal}=4 \cdot 184 \mathrm{~kJ}$.

IIWilcoxon rank-sum test.

Combined occupational, cycling and sports activities.

baseline. Each $100 \mathrm{mg}$ increment of arachidonic acid intake resulted in a 5-year weight gain of $0.62 \%$ and $0.42 \%$ in men and women with normal weight at baseline, respectively. None of the other fatty acids were significantly associated with weight gain in the linear regression models. Total SFA, MUFA or PUFA intake was not associated with weight gain (data not shown). Parameter estimates obtained from the repetition of the analyses using MUFA replacement models were quite similar to those obtained from the carbohydrate replacement models (data not shown).
Results of the multinomial logistic regression analysis for male and female participants are presented in Tables 4 and 5, respectively. As seen in the linear regression models, the risk estimates of the multinomial logistic regression indicate a positive association between stearic acid intake and weight gain, although not all were statistically significant. With regard to the dietary intake of oleic acid, an inverse association was observed in male (Table 4) and female (Table 5) participants who were normal weight at baseline. In both men and women, the dietary intake of linoleic acid ( $n$-6) was positively 
Table 2 Distribution of categories of incident weight change by age groups in male and female participants of the EPIC-Heidelberg cohort

\begin{tabular}{|c|c|c|c|c|c|c|c|c|}
\hline \multirow[b]{2}{*}{ Five-year weight change ${ }^{*}$ category } & \multicolumn{2}{|c|}{$<45$ years } & \multicolumn{2}{|c|}{$45-<55$ years } & \multicolumn{2}{|c|}{$55-65$ years } & \multicolumn{2}{|c|}{ Total } \\
\hline & $\%$ & $n$ & $\%$ & $n$ & $\%$ & $n$ & $\%$ & $n$ \\
\hline \multicolumn{9}{|l|}{ Men ( $n$ 9182) } \\
\hline Weight loss $(<-2.5 \% / 5$ years $)$ & $15 \cdot 2$ & 309 & $16 \cdot 7$ & 629 & $21 \cdot 7$ & 733 & $18 \cdot 2$ & 1671 \\
\hline Stable weight $( \pm 2.5 \% / 5$ years $)$ & $44 \cdot 2$ & 896 & $51 \cdot 4$ & 1942 & $53 \cdot 7$ & 1815 & $50 \cdot 7$ & 4653 \\
\hline Small gain $(+2 \cdot 5 \%$ to $+7 \cdot 5 \% / 5$ years $)$ & $31 \cdot 9$ & 647 & $25 \cdot 2$ & 953 & $20 \cdot 3$ & 685 & $24 \cdot 9$ & 2285 \\
\hline Large gain $(>7.5 \% / 5$ years $)$ & $8 \cdot 6$ & 175 & $6 \cdot 7$ & 252 & $4 \cdot 3$ & 146 & $6 \cdot 2$ & 573 \\
\hline \multicolumn{9}{|l|}{ Women $(n$ 10867) } \\
\hline Weight loss $(<-2.5 \% / 5$ years $)$ & $15 \cdot 9$ & 667 & $17 \cdot 8$ & 648 & $19 \cdot 7$ & 597 & $17 \cdot 6$ & 1912 \\
\hline Stable weight $( \pm 2.5 \% / 5$ years $)$ & $39 \cdot 4$ & 1653 & $42 \cdot 3$ & 1536 & $47 \cdot 6$ & 1447 & $42 \cdot 7$ & 4636 \\
\hline Small gain $(+2 \cdot 5 \%$ to $+7 \cdot 5 \% / 5$ years $)$ & $31 \cdot 0$ & 1301 & $29 \cdot 3$ & 1064 & $25 \cdot 5$ & 774 & $28 \cdot 9$ & 3139 \\
\hline Large gain $(>7.5 \% / 5$ years $)$ & $13 \cdot 7$ & 575 & $10 \cdot 6$ & 385 & $7 \cdot 2$ & 220 & $10 \cdot 9$ & 1180 \\
\hline
\end{tabular}

EPIC-Heidelberg, Heidelberg cohort of the European Prospective Investigation into Cancer and Nutrition.

*Difference of latest follow-up weight minus baseline weight, divided by baseline weight and years of follow-up, and multiplied by five.

Table 3 Association between energy-adjusted dietary fatty acid intake and prospective weight gain in male and female participants of the EPIC-Heidelberg cohort* as assessed by multivariate linear regression models

\begin{tabular}{|c|c|c|c|c|c|c|}
\hline & \multicolumn{3}{|c|}{ All subjects } & \multicolumn{3}{|c|}{ Normal weight subjects at baselinet } \\
\hline & $\beta \ddagger$ & SE & $P$ value & $\beta \ddagger$ & SE & $P$ value \\
\hline Men & \multicolumn{3}{|c|}{$n 7511$} & \multicolumn{3}{|c|}{$n 2550$} \\
\hline \multicolumn{7}{|l|}{ SFA } \\
\hline Palmitic acid $(10 \mathrm{~g} / \mathrm{d}) \S$ & $-1 \cdot 64$ & $1 \cdot 40$ & $0 \cdot 24$ & $-2 \cdot 53$ & $2 \cdot 51$ & $0 \cdot 31$ \\
\hline Stearic acid $(10 \mathrm{~g} / \mathrm{d})$ & $3 \cdot 16$ & 1.09 & 0.004 & $4 \cdot 24$ & $1 \cdot 96$ & 0.03 \\
\hline \multicolumn{7}{|l|}{ MUFA } \\
\hline Oleic acid $(10 \mathrm{~g} / \mathrm{d})$ & -0.02 & 0.24 & 0.95 & $-0 \cdot 22$ & $0 \cdot 40$ & 0.57 \\
\hline \multicolumn{7}{|l|}{ PUFA } \\
\hline \multicolumn{7}{|l|}{$n-6$} \\
\hline Linoleic acid $(10 \mathrm{~g} / \mathrm{d})$ & $0 \cdot 31$ & $0 \cdot 20$ & $0 \cdot 12$ & 0.59 & 0.34 & 0.08 \\
\hline Arachidonic acid $(100 \mathrm{mg} / \mathrm{d})$ & 0.23 & $0 \cdot 13$ & 0.08 & 0.62 & 0.24 & 0.01 \\
\hline \multicolumn{7}{|l|}{$n-3$} \\
\hline $\operatorname{ALA}(1 \mathrm{~g} / \mathrm{d})$ & 0.08 & 0.26 & 0.74 & -0.07 & 0.45 & 0.88 \\
\hline $\mathrm{DHA}+\mathrm{EPA}(100 \mathrm{mg} / \mathrm{d}) \|$ & 0.06 & 0.05 & 0.22 & 0.06 & 0.08 & $0 \cdot 45$ \\
\hline Women & \multicolumn{3}{|c|}{$n 8955$} & \multicolumn{3}{|c|}{$n 5090$} \\
\hline \multicolumn{7}{|l|}{ SFA } \\
\hline Palmitic acid $(10 \mathrm{~g} / \mathrm{d})$ & $-2 \cdot 59$ & $1 \cdot 55$ & 0.09 & $-2 \cdot 70$ & $2 \cdot 05$ & $0 \cdot 19$ \\
\hline Stearic acid $(10 \mathrm{~g} / \mathrm{d})$ & $3 \cdot 61$ & $1 \cdot 14$ & 0.002 & $4 \cdot 07$ & $1 \cdot 49$ & 0.01 \\
\hline \multicolumn{7}{|l|}{ MUFA } \\
\hline Oleic acid $(10 \mathrm{~g} / \mathrm{d})$ & -0.25 & $0 \cdot 24$ & 0.29 & $-0 \cdot 28$ & $0 \cdot 30$ & 0.35 \\
\hline \multirow{2}{*}{\multicolumn{7}{|c|}{$\begin{array}{l}\text { PUFA } \\
n-6\end{array}$}} \\
\hline & & & & & & \\
\hline Linoleic acid $(10 \mathrm{~g} / \mathrm{d})$ & 0.62 & $0 \cdot 19$ & 0.00 & $0 \cdot 71$ & 0.25 & 0.00 \\
\hline Arachidonic acid $(100 \mathrm{mg} / \mathrm{d})$ & 0.28 & $0 \cdot 14$ & 0.04 & 0.42 & $0 \cdot 18$ & 0.02 \\
\hline \multicolumn{7}{|l|}{$n-3$} \\
\hline $\operatorname{ALA}(1 \mathrm{~g} / \mathrm{d})$ & -0.03 & 0.26 & 0.91 & $-0 \cdot 19$ & 0.34 & 0.57 \\
\hline DHA + ËPAll $(100 \mathrm{mg} / \mathrm{d})$ & -0.06 & 0.05 & 0.23 & -0.01 & 0.06 & 0.83 \\
\hline
\end{tabular}

EPIC-Heidelberg, Heidelberg cohort of the European Prospective Investigation into Cancer and Nutrition; ALA, $\alpha$-linolenic acid.

${ }^{*}$ Subjects with weight loss ( $<-2.5 \% / 5$ years, $n 1671$ in men, $n 1912$ in women) excluded.

tBaseline BMI between 18.5 and $25 \mathrm{~kg} / \mathrm{m}^{2}$.

$\ddagger$ Adjusted for other PUFA, MUFA and SFA intakes $(\mathrm{g} / \mathrm{d})$, protein, alcohol intake $(\mathrm{g} / \mathrm{d})$, total energy intake $(\mathrm{kcal} / \mathrm{d}(1 \mathrm{kcal}=4 \cdot 184 \mathrm{~kJ}))$, baseline weight $(\mathrm{kg})$ and height $(\mathrm{cm})$, age at baseline (years), physical activity, education, smoking (never, habitual, new, quit), follow-up-time (years); in women aditionally adjusted for menopausal status at baseline (premenopausal, perimenopausal, postmenopausal, surgical menopausal, missing).

$\S$ All nutrients energy-adjusted by the residual method.

IIEntered as sum because of high correlation ( $r>0.97)$.

associated with small and large weight gain. Arachidonic acid intake was positively associated with weight gain in women only (Table 5). Dietary intake of the $n$-3 PUFA ALA was significantly inversely associated with large weight gain in women (Table 5), but not in men (Table 4). The results for EPA and DHA were conflicting; although a positive association was seen in men (Table 4), women in the second tertile of dietary intake had a significantly reduced risk of small weight gain (Table 5). When tertiles of the total intake of SFA, MUFA and PUFA were analysed, only the high intake of PUFA was significantly positively associated with small and large weight gain in men and women (data not shown). Re-evaluation of the analyses with the MUFA replacement model did not alter the 
Table 4 Odds ratios and $95 \%$ confidence intervals for the association between energy-adjusted dietary fatty acid intake (in tertiles) and small and large weight gain ( $v$. stable weight) in male participants of the EPIC-Heidelberg cohort as assessed by multinomial logistic regression models

\begin{tabular}{|c|c|c|c|c|c|c|c|c|}
\hline \multirow[b]{3}{*}{ Men } & \multicolumn{4}{|c|}{ All $(n 7511)$} & \multicolumn{4}{|c|}{ Normal weight subjects at baseline ${ }^{\star}(n 2550)$} \\
\hline & \multicolumn{2}{|c|}{ Small weight gain ( $n$ 2285) } & \multicolumn{2}{|c|}{ Large weight gain $(n 573)$} & \multicolumn{2}{|c|}{ Small weight gain $(n 806)$} & \multicolumn{2}{|c|}{ Large weight gain $(n$ 251) } \\
\hline & OR & $95 \% \mathrm{Cl}+$ & OR & $95 \% \mathrm{Cl}+$ & OR & $95 \% \mathrm{Cl}+$ & OR & $95 \% \mathrm{Cl}+$ \\
\hline \multicolumn{9}{|c|}{ SFA } \\
\hline \multicolumn{9}{|c|}{ Palmitic acid $(\mathrm{g} / \mathrm{d}) \ddagger$} \\
\hline$<12 \cdot 8$ & $1 \cdot 00$ & & $1 \cdot 00$ & & $1 \cdot 00$ & & $1 \cdot 00$ & \\
\hline $12 \cdot 8-15 \cdot 1$ & $1 \cdot 17$ & $0.94,1.47$ & $1 \cdot 33$ & $0.89,1.97$ & 0.95 & $0 \cdot 64,1 \cdot 40$ & $1 \cdot 14$ & $0 \cdot 62,2 \cdot 13$ \\
\hline$>15 \cdot 1$ & $1 \cdot 27$ & $0.91,1.75$ & $1 \cdot 40$ & $0 \cdot 79,2 \cdot 48$ & $1 \cdot 01$ & $0.58,1.78$ & $1 \cdot 11$ & $0 \cdot 46,2 \cdot 70$ \\
\hline \multirow{2}{*}{\multicolumn{9}{|c|}{ Stearic acid $(\mathrm{g} / \mathrm{d})$}} \\
\hline & & & & & & & & \\
\hline$<5 \cdot 5$ & $1 \cdot 00$ & & $1 \cdot 00$ & & $1 \cdot 00$ & & $1 \cdot 00$ & \\
\hline $5 \cdot 5-6 \cdot 6$ & 0.85 & $0 \cdot 70,1 \cdot 02$ & 0.92 & $0 \cdot 66,1 \cdot 28$ & 0.99 & $0 \cdot 72,1 \cdot 35$ & $1 \cdot 23$ & $0.74,2.03$ \\
\hline$>6 \cdot 6$ & 1.06 & $0 \cdot 83,1 \cdot 35$ & $1 \cdot 25$ & $0.82,1.91$ & $1 \cdot 21$ & $0 \cdot 80,1 \cdot 82$ & $1 \cdot 85$ & $0.98,3.48$ \\
\hline \multirow{2}{*}{\multicolumn{9}{|c|}{$\begin{array}{l}\text { MUFA } \\
\text { trend }\end{array}$}} \\
\hline & & & & & & & & \\
\hline \multicolumn{9}{|c|}{ Oleic acid $(g / d)$} \\
\hline$<20.00$ & $1 \cdot 00$ & & $1 \cdot 00$ & & $1 \cdot 00$ & & $1 \cdot 00$ & \\
\hline $20 \cdot 0-23 \cdot 2$ & 0.98 & $0.83,1 \cdot 16$ & $1 \cdot 12$ & $0.83,1.51$ & $0 \cdot 89$ & $0 \cdot 67,1 \cdot 18$ & 0.87 & $0.56,1.38$ \\
\hline$>23 \cdot 2$ & 0.89 & $0 \cdot 71,1 \cdot 11$ & 0.93 & $0.63,1.37$ & 0.82 & $0.57,1 \cdot 17$ & 0.56 & $0.31,1.00$ \\
\hline$P_{\text {trend }}$ & 0.32 & & 0.66 & & 0.29 & & 0.04 & \\
\hline PUFA & & & & & & & & \\
\hline \multicolumn{9}{|c|}{$n-6$} \\
\hline \multicolumn{9}{|c|}{ Linoleic acid $(\mathrm{g} / \mathrm{d})$} \\
\hline$<8.6$ & 1.00 & & $1 \cdot 00$ & & $1 \cdot 00$ & & $1 \cdot 00$ & \\
\hline $8 \cdot 6-10 \cdot 9$ & $1 \cdot 11$ & $0.97,1 \cdot 28$ & 1.09 & $0 \cdot 85,1 \cdot 40$ & $1 \cdot 03$ & $0 \cdot 81,1 \cdot 30$ & $1 \cdot 23$ & $0 \cdot 83,1 \cdot 82$ \\
\hline$>10 \cdot 9$ & $1 \cdot 18$ & $1 \cdot 00,1.39$ & $1 \cdot 27$ & $0.94,1.70$ & $1 \cdot 19$ & $0.90,1.58$ & 1.64 & $1 \cdot 04,2 \cdot 61$ \\
\hline$P_{\text {trend }}$ & 0.08 & & $0 \cdot 11$ & & 0.22 & & 0.03 & \\
\hline \multicolumn{9}{|c|}{ Arachidonic acid $(\mathrm{g} / \mathrm{d})$} \\
\hline$<0 \cdot 14$ & $1 \cdot 00$ & & $1 \cdot 00$ & & $1 \cdot 00$ & & $1 \cdot 00$ & \\
\hline $0 \cdot 14-0 \cdot 19$ & $1 \cdot 01$ & $0 \cdot 87,1 \cdot 17$ & $0 \cdot 82$ & $0.62,1.07$ & $1 \cdot 09$ & $0 \cdot 84,1 \cdot 40$ & 0.91 & $0 \cdot 60,1 \cdot 36$ \\
\hline$>0 \cdot 19$ & 0.97 & $0 \cdot 80,1 \cdot 17$ & $0 \cdot 81$ & $0.58,1.14$ & 1.00 & $0.71,1 \cdot 39$ & 0.85 & $0.49,1.46$ \\
\hline$P_{\text {trend }}$ & 0.64 & & $0 \cdot 27$ & & 0.95 & & 0.58 & \\
\hline \multicolumn{9}{|l|}{$n-3$} \\
\hline \multicolumn{9}{|l|}{ ALA $(g / d)$} \\
\hline$<1.04$ & $1 \cdot 00$ & & $1 \cdot 00$ & & $1 \cdot 00$ & & 1.00 & \\
\hline $1 \cdot 04-1 \cdot 23$ & 0.95 & $0 \cdot 82,1 \cdot 10$ & 0.95 & $0.73,1.24$ & $1 \cdot 11$ & $0.85,1.44$ & 0.88 & $0.57,1.35$ \\
\hline$>1 \cdot 23$ & 0.97 & $0 \cdot 81,1 \cdot 16$ & $1 \cdot 12$ & $0.83,1.53$ & $1 \cdot 07$ & $0.78,1 \cdot 46$ & 0.86 & $0.52,1.43$ \\
\hline$P_{\text {trend }}$ & $0 \cdot 82$ & & 0.36 & & 0.79 & & 0.62 & \\
\hline $\begin{array}{l}\mathrm{EPA}+\mathrm{DHA}( \\
<0 \cdot 16\end{array}$ & $1 \cdot 00$ & & $1 \cdot 00$ & & $1 \cdot 00$ & & $1 \cdot 00$ & \\
\hline $0.16-0.28$ & 1.06 & $0.92,1.21$ & $1 \cdot 23$ & $0.97,1.57$ & $1 \cdot 17$ & $0.93,1.47$ & $1 \cdot 25$ & $0 \cdot 86,1 \cdot 82$ \\
\hline$>0.28$ & $1 \cdot 17$ & $0.99,1.37$ & 1.43 & $1.07,1.90$ & 1.03 & $0.78,1.36$ & $1 \cdot 34$ & $0 \cdot 85,2 \cdot 12$ \\
\hline$P_{\text {trend }}$ & 0.05 & & 0.02 & & 0.92 & & 0.22 & \\
\hline
\end{tabular}

EPIC-Heidelberg, Heidelberg cohort of the European Prospective Investigation into Cancer and Nutrition; ALA, $\alpha$-linolenic acid.

${ }^{*}$ Baseline BMI between 18.5 and $25 \mathrm{~kg} / \mathrm{m}^{2}$.

tAdjusted for other PUFA, MUFA, SFA intakes, protein, alcohol intake, total energy intake, baseline weight (kg) and height (cm), age at baseline (years), physical activity, education, smoking (never, habitual, new, quit), follow-up time (years) and menopausal status in women.

$\ddagger$ All nutrients energy-adjusted by the residual method.

$\S$ Entered as sum because of high correlation $(r>0.97)$.

Bold values are estimates significant at $P<0.05$.

risk estimates substantially (data not shown). The OR obtained differed from those from the carbohydrate replacement models by the second decimal only. In terms of weight loss (data not shown), in the case of arachidonic acid, positive associations with weight gain were reflected by the tendency of inverse associations with weight loss (OR for weight loss, comparing highest $v$. lowest tertile, was $0.80(95 \%$ CI $0.64,0.99)$ in men and 0.85 (95\% CI $0.69,1.04)$ in women). Dietary intake of ALA, palmitic, stearic and acids was not associated with weight loss.
The associations between fatty acid intake and incident overweight and obesity, respectively, are shown in Fig. 1. As in the multinomial logistic regression, stearic acid was positively associated with weight gain. Compared with participants in the lowest tertile, men in the highest tertile of stearic acid intake had a significantly increased OR of becoming overweight, while women in the same tertile had a significantly increased OR of becoming incident obese. The n-6 PUFA of linoleic and arachidonic acids were positively associated with incident overweight and obesity, especially in women. With regard to ALA, non-significant 
Table 5 Odds ratios and $95 \%$ confidence intervals for the association between energy-adjusted dietary fatty acid intake (in tertiles) and small and large weight gain ( $v$. stable weight) in female participants of the EPIC-Heidelberg cohort as assessed by multinomial logistic regression models

\begin{tabular}{|c|c|c|c|c|c|c|c|c|}
\hline \multirow[b]{3}{*}{ Women } & \multicolumn{4}{|c|}{ All $(n$ 8955) } & \multicolumn{4}{|c|}{ Normal weight subjects at baseline ${ }^{\star}(n 5090)$} \\
\hline & \multicolumn{2}{|c|}{ Small weight gain ( $n$ 3139) } & \multicolumn{2}{|c|}{ Large weight gain $(n 1180)$} & \multicolumn{2}{|c|}{ Small weight gain ( $n$ 1689) } & \multicolumn{2}{|c|}{ Large weight gain $(n 581)$} \\
\hline & OR & $95 \% \mathrm{Cl}+$ & OR & $95 \% \mathrm{Cl}+$ & OR & $95 \% \mathrm{Clt}$ & OR & $95 \% \mathrm{Cl}+$ \\
\hline \multicolumn{9}{|c|}{ SFA } \\
\hline \multicolumn{9}{|c|}{ Palmitic acid $(\mathrm{g} / \mathrm{d}) \ddagger$} \\
\hline$<12 \cdot 8$ & $1 \cdot 00$ & & $1 \cdot 00$ & & $1 \cdot 00$ & & $1 \cdot 00$ & \\
\hline $12 \cdot 8-15 \cdot 1$ & 1.00 & $0 \cdot 82,1 \cdot 21$ & $1 \cdot 07$ & $0 \cdot 81,1 \cdot 42$ & $1 \cdot 06$ & $0 \cdot 82,1 \cdot 38$ & $1 \cdot 17$ & $0 \cdot 79,1 \cdot 72$ \\
\hline$>15 \cdot 1$ & 0.93 & $0 \cdot 70,1 \cdot 24$ & $1 \cdot 01$ & $0.67,1.52$ & $1 \cdot 29$ & $0 \cdot 88,1 \cdot 89$ & $1 \cdot 23$ & $0 \cdot 69,2 \cdot 17$ \\
\hline$P_{\text {trend }}$ & 0.56 & & 0.99 & & $0 \cdot 18$ & & 0.52 & \\
\hline \multicolumn{9}{|c|}{$\begin{array}{l}r \text { trend } \\
\text { Stearic acid }(\mathrm{g} / \mathrm{d})\end{array}$} \\
\hline$<5.5$ & $1 \cdot 00$ & & $1 \cdot 00$ & & $1 \cdot 00$ & & 1.00 & \\
\hline $5 \cdot 5-6 \cdot 6$ & $1 \cdot 07$ & $0 \cdot 92,1 \cdot 26$ & $1 \cdot 26$ & $0.99,1.59$ & $1 \cdot 07$ & $0 \cdot 86,1 \cdot 32$ & $1 \cdot 26$ & $0.91,1 \cdot 74$ \\
\hline$>6 \cdot 6$ & $1 \cdot 16$ & $0.94,1.43$ & $1 \cdot 39$ & $1 \cdot 03,1 \cdot 88$ & 1.08 & $0.82,1.42$ & $1 \cdot 50$ & $1 \cdot 00,2 \cdot 26$ \\
\hline \multirow{2}{*}{\multicolumn{9}{|c|}{$\begin{array}{l}\text { MUFA } \\
\text { MUend }\end{array}$}} \\
\hline & & & & & & & & \\
\hline \multicolumn{9}{|c|}{ Oleic acid (g/d) } \\
\hline$<20.5$ & $1 \cdot 00$ & & $1 \cdot 00$ & & 1.00 & & $1 \cdot 00$ & \\
\hline $20 \cdot 5-23 \cdot 8$ & 0.91 & $0.79,1.05$ & 0.95 & $0 \cdot 77,1 \cdot 17$ & $0 \cdot 86$ & $0.71,1.03$ & 0.93 & $0 \cdot 70,1 \cdot 24$ \\
\hline$>23 \cdot 8$ & $0 \cdot 86$ & $0.71,1.02$ & 0.89 & $0 \cdot 69,1 \cdot 16$ & 0.76 & $0.60,0.96$ & $0 \cdot 78$ & $0 \cdot 55,1 \cdot 10$ \\
\hline $\begin{array}{l}P_{\text {trend }} \\
\text { PUFA }\end{array}$ & $0 \cdot 10$ & & $0 \cdot 37$ & & 0.02 & & $0 \cdot 14$ & \\
\hline \multicolumn{9}{|l|}{$\begin{array}{l}\text { PUFA } \\
n-6\end{array}$} \\
\hline \multicolumn{9}{|c|}{ Linoleic acid $(\mathrm{g} / \mathrm{d})$} \\
\hline$<8.9$ & $1 \cdot 00$ & & $1 \cdot 00$ & & $1 \cdot 00$ & & $1 \cdot 00$ & \\
\hline $8 \cdot 9-11 \cdot 2$ & $1 \cdot 06$ & $0 \cdot 94,1 \cdot 20$ & $1 \cdot 10$ & $0 \cdot 92,1 \cdot 32$ & $1 \cdot 16$ & $0.99,1.36$ & $1 \cdot 12$ & $0 \cdot 88,1 \cdot 43$ \\
\hline$>11 \cdot 2$ & $1 \cdot 24$ & $1.08,1.43$ & 1.56 & $1 \cdot 27,1 \cdot 90$ & $1 \cdot 36$ & $1 \cdot 13,1 \cdot 64$ & 1.65 & $1 \cdot 25,2 \cdot 18$ \\
\hline$P_{\text {trend }}$ & $0 \cdot 00$ & & $<0.00$ & & 0.00 & & $0 \cdot 00$ & \\
\hline \multicolumn{9}{|c|}{ Arachidonic acid (g/d) } \\
\hline$<0.12$ & $1 \cdot 00$ & & $1 \cdot 00$ & & $1 \cdot 00$ & & $1 \cdot 00$ & \\
\hline $0 \cdot 12-0.17$ & $1 \cdot 16$ & $1 \cdot 02,1 \cdot 33$ & $1 \cdot 10$ & $0.90,1.35$ & 1.23 & $1 \cdot 04,1 \cdot 47$ & 0.92 & $0 \cdot 70,1 \cdot 21$ \\
\hline$>0 \cdot 17$ & $1 \cdot 30$ & $1 \cdot 10,1 \cdot 54$ & $1 \cdot 23$ & $0.96,1.58$ & $1 \cdot 40$ & $1 \cdot 11,1 \cdot 76$ & $1 \cdot 15$ & $0.81,1.62$ \\
\hline$P_{\text {trend }}$ & 0.01 & & $0 \cdot 16$ & & 0.01 & & 0.50 & \\
\hline \multirow{2}{*}{\multicolumn{9}{|c|}{ ALA $(\mathrm{g} / \mathrm{d})$}} \\
\hline & & & & & & & & \\
\hline$<1 \cdot 1$ & $1 \cdot 00$ & & $1 \cdot 00$ & & $1 \cdot 00$ & & $1 \cdot 00$ & \\
\hline $1 \cdot 1-1 \cdot 3$ & $0 \cdot 88$ & $0 \cdot 78,1 \cdot 00$ & 0.85 & $0 \cdot 71,1 \cdot 02$ & 0.90 & $0 \cdot 77,1 \cdot 07$ & $0 \cdot 88$ & $0 \cdot 68,1 \cdot 13$ \\
\hline$>1 \cdot 3$ & $0 \cdot 88$ & $0 \cdot 76,1 \cdot 02$ & 0.80 & $0.65,0.99$ & $0 \cdot 86$ & $0 \cdot 71,1 \cdot 04$ & $0 \cdot 82$ & $0 \cdot 62,1 \cdot 10$ \\
\hline$P_{\text {trend }}$ & $0 \cdot 11$ & & 0.05 & & $0 \cdot 14$ & & 0.20 & \\
\hline \multicolumn{9}{|c|}{$\mathrm{EPA}+\mathrm{DHA}(\mathrm{g} / \mathrm{d}) \S$} \\
\hline$<0.15$ & $1 \cdot 00$ & & $1 \cdot 00$ & & $1 \cdot 00$ & & $1 \cdot 00$ & \\
\hline $0.15-0.25$ & 0.81 & $0.72,0.92$ & 0.87 & $0.72,1.03$ & 0.82 & $0.70,0.97$ & 0.83 & $0.65,1.06$ \\
\hline$>0.25$ & 0.90 & $0.78,1.05$ & 0.94 & $0 \cdot 76,1 \cdot 16$ & 0.89 & $0.73,1.08$ & 0.97 & $0 \cdot 72,1 \cdot 30$ \\
\hline$P_{\text {trend }}$ & $0 \cdot 40$ & & $0 \cdot 73$ & & 0.36 & & 0.93 & \\
\hline
\end{tabular}

EPIC-Heidelberg, Heidelberg cohort of the European Prospective Investigation into Cancer and Nutrition; ALA, $\alpha$-linolenic acid.

${ }^{*}$ Baseline BMI between 18.5 and $25 \mathrm{~kg} / \mathrm{m}^{2}$.

tAdjusted for other PUFA, MUFA, SFA intakes, protein, alcohol intake, total energy intake, baseline weight (kg) and height (cm), age at baseline (years), physical activity, education, smoking (never, habitual, new, quit), follow-up time (years) and menopausal status in women.

$\ddagger$ All nutrients energy-adjusted by the residual method.

$\S$ Entered as sum because of high correlation $(r>0.97)$.

Bold values are estimates significant at $P<0.05$.

inverse associations between incident overweight and obesity were observed in both men and women.

\section{Discussion}

In the present study, the effects of dietary fatty acid intakes on long-term weight change were investigated in a prospective setting. Weight-promoting effects were observed for stearic, linoleic and arachidonic acids in men and women, whereas ALA showed inverse associations with weight gain in women. Oleic acid intake was inversely associated with prospective weight gain in the multinomial logistic regression, especially after restriction to participants who were normal weight at baseline. No clear associations were observed for palmitic acid and the sum of EPA and DHA.

Several biological mechanisms may help in explaining the differential effects of fatty acids on weight development in adults, as observed in the present study. Fatty acids differ slightly with respect to their energy content. The order of fatty acids according to their energy content is linolenic acid $(38.7 \mathrm{~kJ} / \mathrm{g})<$ linoleic $(39 \cdot 0 \mathrm{~kJ} / \mathrm{g})<$ palmitic acid $(39 \cdot 2 \mathrm{~kJ} / \mathrm{g})<$ oleic $(39 \cdot 4 \mathrm{~kJ} / \mathrm{g})<$ stearic acid 

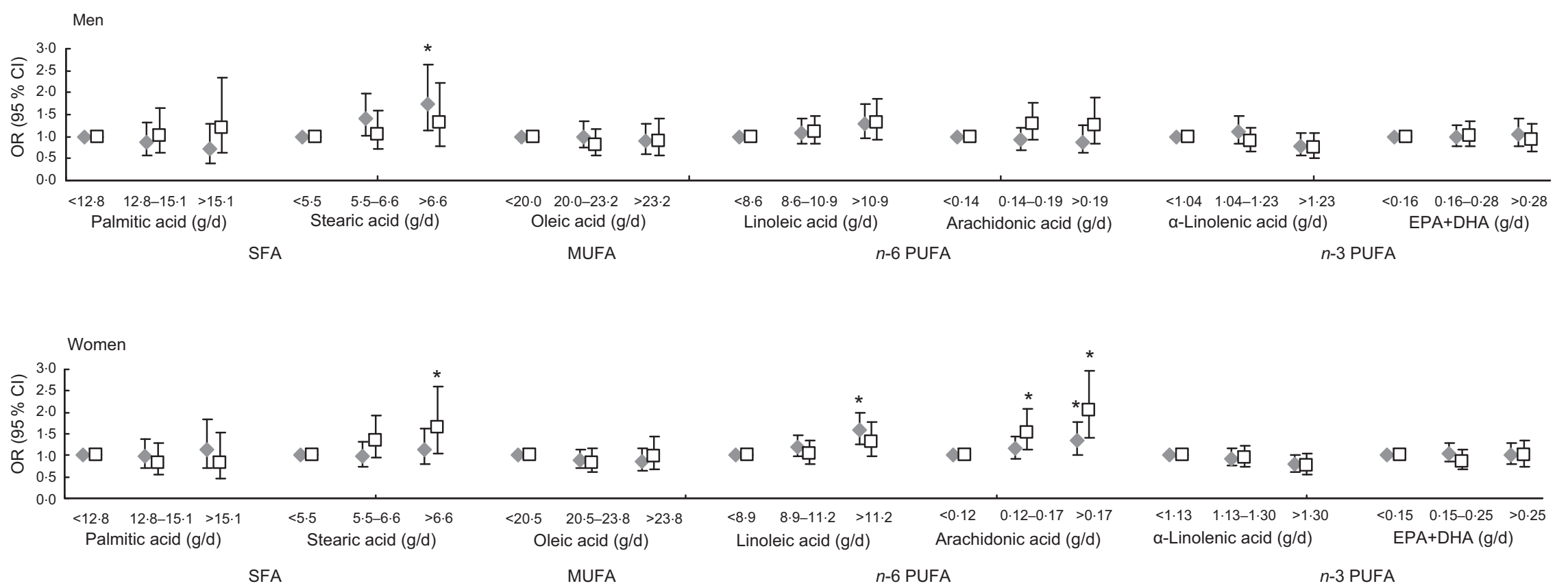

Fig. 1 Multivariate adjusted OR (bars indicate $95 \% \mathrm{Cl}$ ) for the association between fatty acid intake and incident overweight (๑) or obesity ( $\square$ ), respectively. ${ }^{*} P_{\text {trend }}<0 \cdot 01$ 
$(40 \cdot 0 \mathrm{~kJ} / \mathrm{g})<$ arachidonic acid $(40 \cdot 5 \mathrm{~kJ} / \mathrm{g})^{(23)}$. However, the metabolisable energy depends on the intestinal absorption, which is lower for stearic acid than for other fatty acids such as palmitic, oleic or linoleic acid ${ }^{(24,25)}$. Considering that some fatty acids are consumed in very small amounts, differences in energy content and bioavailability cannot be the only explanation for the observed effects. Furthermore, in the present study, dietary fatty acid intake was adjusted for energy intake, so that differences in energy content are rather unlikely to explain the observed results. A diet with a high proportion of PUFA has been shown to induce a higher thermogenic effect in man than a diet high in SFA ${ }^{(10)}$. The oxidation rates of fatty acids depend on the chain length and the degree of saturation. In man, the order of oxidation rates from highest to lowest was observed as follows: ALA $>$ linoleic acid $>$ oleic acid $>$ palmitoleic acid $>$ stearic acid ${ }^{(12)}$. Accordingly, a diet high in saturated fats (such as stearic acid) with a low oxidation rate might be more prone to lead to weight gain than a diet rich in polyunsaturated fats (such as ALA) with a higher oxidation rate. In a study on rats, animals on a diet high in beef tallow (saturated fat) accumulated more body fat than animals on a diet high in safflower oil (polyunsaturated fat), which was related to a decrease in sympathetic activity in the beef tallow diet ${ }^{(13)}$. In addition to effects directly related to the efficiency of energy storage, certain fatty acids, (i.e. $n-3$ and $n$-6 PUFA) have been shown to act as transcription factors influencing the expression of genes important in fat metabolism. Especially, $n$ - 3 fatty acids (ALA, EPA and DHA) promote the expression of genes involved in fat oxidation and thermogenesis, yielding decreased body fat deposition and improved glucose clearance ${ }^{(16)}$. Fatty acids may also exert different effects on postprandial appetite. In an experimental study on normal weight men, food intake was lowest after intestinal infusion of linoleic acid than that of oleic or stearic acid ${ }^{(26)}$. In contrast, in a study in which test meals with different fatty acid compositions were given to overweight men, no differences regarding subsequent energy intake were observed ${ }^{(27)}$.

In the present study, dietary intake of stearic acid was positively associated with weight gain, especially in the linear regression approach. This finding seems plausible in the context of the low oxidation rate (i.e. fat is more prone to be stored in fat tissue) and relatively high energy content of stearic $\operatorname{acid}^{(12)}$. However, no association was found for palmitic acid, which has an oxidation rate only slightly above stearic acid and provides minimally lower energy content. Neither prospective nor cross-sectional analyses have been conducted in order to explicitly investigate the effect of habitual intake of these two SFA on BMI or weight gain.

Oleic acid, contributing $90 \%$ to total MUFA intake, was inversely associated with prospective weight gain in the multinomial logistic regression restricted to participants who were normal weight at baseline. There are no directly comparable studies investigating oleic acid intake and weight change prospectively. In a prospective cohort study from Spain (SUN study), baseline consumption of olive oil (70\% oleic acid) was associated with a lower likelihood of weight gain, although not statistically significant ${ }^{(28)}$. The comparability of Bes-Rastrollo et al.'s ${ }^{(28)}$ study to the present study might be impaired due to differing dietary habits in Spain and Germany, especially in terms of olive oil consumption, which determine high intake of oleic acid.

The n-6 PUFA linoleic and arachidonic acids showed weight-promoting effects in the linear regression as well as in the multinomial logistic regression models. In the Nurses' Health Study, linoleic acid intake was positively correlated with $\mathrm{BMI}^{(15)}$. To our knowledge, the effect of dietary arachidonic acid intake on prospective weight change has not been investigated in an epidemiological study so far. However, in the EPIC-Potsdam study, dietary intake of meat, the major food source of arachidonic acid, was associated with increased risk of a large, 2-year weight gain in women ${ }^{(29)}$.

Reduced risks of small and large weight gain were observed in women with high ALA intake, significantly so for large weight gain. Although an inverse association between dietary intake of ALA and weight gain has not been observed in an epidemiological study so far, this finding seems plausible in the context of the high oxidation rate of ALA and its potential activity as a transcription factor enhancing fat oxidation rates and thermogenesis.

Here, the applied isoenergetic models represent the effect of substituting energy from carbohydrates by the same amount of energy provided by a certain fatty acid (carbohydrate replacement model). Increased intake of a certain fatty acid in such a model implies the proportion of energy provided by fat to increase. A high percentage of energy from fat has been associated with weight gain in several epidemiological studies ${ }^{(30)}$. In this context, increased intake of any fatty acid in the carbohydrate replacement model would be expected to promote weight gain due to the implied higher proportion of energy from fat. However, the quite similar results obtained from the MUFA replacement models show that certain fatty acids also affect weight change when the percentage of energy from fat remains constant (i.e. substituting energy from MUFA by energy from the fatty acid of interest).

The linear and the multinomial logistic regression approaches yielded fairly consistent results. The definition of incident overweight/obesity is an alternative approach to study weight change longitudinally, with a more healthrelated focus. The consistent results of the analysis of incident overweight/obesity, as compared to weight gain per se, argue for a factor associated with weight gain to be most likely also related to incident overweight or obesity.

Apart from the fact that our findings require confirmation by other studies, the conclusion in terms of 
dietary advice for the prevention of weight gain by controlled fatty acid intake would include a reduced consumption of meat and dairy products (low intake of arachidonic and stearic acids) and a higher consumption of plant food rich in ALA (e.g. nuts or rapeseed oil) ${ }^{(31)}$. Such advice would be in agreement with existing dietary guidelines for the prevention of chronic disease occurrence.

\section{Limitations}

Several limitations of the present study should be noted. First, the ability to assess absolute intake of individual dietary fatty acids by means of an FFQ is limited. Furthermore, by using dietary intake data ascertained by FFQ at baseline in this prospective analysis, we assume that dietary habits remain stable over time. This assumption, however, seems justified, as moderate-to-good long-term reproducibility of the FFQ data and fair agreement of individual classification by food groups and nutrients between baseline and follow-up were observed in a comparison of the two dietary assessments in our study population ${ }^{(35)}$. The validity of fat intake assessment by the EPIC-Heidelberg FFQ has been evaluated by using twelve $24 \mathrm{~h}$ dietary recalls as a reference method. The resulting adjusted correlation coefficients were $0.75,0.51$ and 0.43 for PUFA, MUFA and SFA, respectively ${ }^{(17)}$. However, the validity of specific fatty acid intake has not been addressed in the present validation study. Thus, it can only be assumed that the validity of specific fatty acid data is similar to that observed in subgroups of comparable cohort studies by using weighed dietary records ${ }^{(32-34)}$ or subcutaneous fat aspirates ${ }^{(34)}$ as reference methods. In a British validation study of an FFQ that included a greater detail of foods from which the majority of dietary fatty acids are obtained, correlation coefficients of energyadjusted fatty acid intake with $7 \mathrm{~d}$ weighed records were 0.77 for palmitic, 0.70 for stearic, 0.20 for oleic, 0.24 for linoleic and $0 \cdot 70$ for arachidonic acids ${ }^{(32)}$. Similarly, in a Japanese validation study of a 138 -item FFQ with $28 \mathrm{~d}$ weighed records, relatively high correlations were observed for stearic and palmitic acids $(0.61$ and 0.63 , respectively), whereas correlation coefficients for PUFA were rather low, between $0 \cdot 27$ (linolenic acid) and 0.38 $(\mathrm{EPA})^{(33)}$. Overall, it cannot be dismissed that the fatty acid intake data in the present study require cautious interpretation, also because databases on the fatty acid composition of food are limited in precision. Nevertheless, we hope that the results of this hypothesis-driven analysis stimulate further research on this question, ideally involving studies applying more precise methods of dietary fatty acid intake measurement.

Also, the validity and precision of the self-reported body weight at follow-up are limited. However, the selfreported data were calibrated by a method using BMIand sex-specific equations derived from a $7 \%$ subsample to reduce the misclassification error.
It has been shown that the accuracy of weight ${ }^{(36)}$ and $\operatorname{diet}^{(37)}$ reports depends on BMI. Therefore, the results of the subanalysis restricted to participants with normal weight at baseline may be regarded as more reliable than the analysis of the total cohort. Results from the full analysis and the subanalysis were, however, fairly comparable.

Here, the applied index ranking participants in terms of their physical activity combining occupational and leisure-time physical activity has been validated using accelerometers $^{(38)}$ and heart rate monitoring ${ }^{(20)}$ as reference instruments, showing acceptable agreement. Nevertheless, a more precise instrument to measure physical activity would have been desirable.

\section{Conclusion}

Here, the observed associations of fatty acid intake with prospective weight change lack comparable analyses from other prospective studies, and even experimental studies on human subjects focusing on specific fatty acids and their effect on weight change are scarce. Therefore, further studies on this issue are strongly warranted before detailed dietary recommendations focussing on the prevention of adult weight gain can be given.

\section{Acknowledgements}

The present study was supported by funds of the KurtEberhard-Bode-Stiftung. K.N. was the recipient of a scholarship from the Deutsche Forschungsgemeinschaft, Graduiertenkolleg 793. K.N. was responsible for the statistical analysis and interpretation of the data and drafting the manuscript. G.B.B. was responsible for critical revision of the manuscript for important intellectual content. J.L. was responsible for the study concept and design, acquisition of data, critical revision of the manuscript for important intellectual content, and for obtaining the funding for the study. None of the authors had any conflicts of interest in connection with the present study.

\section{References}

1. Helmert U \& Strube H (2004) The development of obesity in Germany in the period from 1985 until 2000. Gesundbeitswesen 66, 409-415.

2. James PT (2004) Obesity: the worldwide epidemic. Clin Dermatol 22, 276-280.

3. Sherwood NE, Jeffery RW, French SA et al. (2000) Predictors of weight gain in the Pound of Prevention Study. Int J Obes Relat Metab Disord 24, 395-403.

4. Bergmann MM \& Mensink GB (1999) Anthropometric data and obesity. Gesundheitswesen 61, S115-SS20.

5. Boeing H (2005) Macht Fett wirklich fett? Ernhrungsumschau 52, 4-8.

6. Kasper H (2000) Adipositas. Ernaebrungsmedizin und Diaetetik, pp. 241-263. Jena: Urban \& Fischer.

7. Kroke A, Liese AD, Schulz M et al. (2002) Recent weight changes and weight cycling as predictors of subsequent 
two year weight change in a middle-aged cohort. Int J Obes Relat Metab Disord 26, 403-409.

8. Doucet E, Almeras N, White MD et al. (1998) Dietary fat composition and human adiposity. Eur J Clin Nutr 52, 2-6.

9. Astrup A (2005) The role of dietary fat in obesity. Semin Vasc Med 5, 40-47.

10. Marken Lichtenbelt WD, Mensink RP \& Westerterp KR (1997) The effect of fat composition of the diet on energy metabolism. Z Ernahrungswiss 36, 303-305.

11. Kien CL, Bunn JY \& Ugrasbul F (2005) Increasing dietary palmitic acid decreases fat oxidation and daily energy expenditure. Am J Clin Nutr 82, 320-326.

12. DeLany JP, Windhauser MM, Champagne CM et al. (2000) Differential oxidation of individual dietary fatty acids in humans. Am J Clin Nutr 72, 905-911.

13. Matsuo T, Shimomura Y, Saitoh S et al. (1995) Sympathetic activity is lower in rats fed a beef tallow diet than in rats fed a safflower oil diet. Metabolism 44, 934-939.

14. Gonzalez CA, Pera G, Quiros JR et al. (2000) Types of fat intake and body mass index in a Mediterranean country. Public Health Nutr 3, 329-336.

15. Colditz GA, Willett WC, Stampfer MJ et al. (1990) Patterns of weight change and their relation to diet in a cohort of healthy women. Am J Clin Nutr 51, 1100-1105.

16. Clarke SD (2000) Polyunsaturated fatty acid regulation of gene transcription: a mechanism to improve energy balance and insulin resistance. Br J Nutr 83, Suppl. 1, S59-S66.

17. Bohlscheid-Thomas S, Hoting I, Boeing H et al. (1997) Reproducibility and relative validity of energy and macronutrient intake of a food frequency questionnaire developed for the German part of the EPIC project. European Prospective Investigation into Cancer and Nutrition. Int J Epidemiol 26, Suppl. 1, S71-S81.

18. Dehne LI, Klemm C, Henseler G et al. (1999) The German Food Code and Nutrient Data Base (BLS II.2). Eur J Epidemiol 15, 355-359.

19. Spencer EA, Appleby PN, Davey GK et al. (2002) Validity of self-reported height and weight in 4808 EPIC-Oxford participants. Public Health Nutr 5, 561-565.

20. Wareham NJ, Jakes RW, Rennie KL et al. (2003) Validity and repeatability of a simple index derived from the short physical activity questionnaire used in the European Prospective Investigation into Cancer and Nutrition (EPIC) study. Public Health Nutr 6, 407-413.

21. Willett W \& Stampfer MJ (1986) Total energy intake: implications for epidemiologic analyses. Am J Epidemiol 124, $17-27$.

22. Kipnis V, Freedman LS, Brown CC et al. (1993) Interpretation of energy adjustment models for nutritional epidemiology. Am J Epidemiol 137, 1376-1380.

23. Elmadfa I \& Leitzmann C (1990) Ernaebrung des Menschen. Stuttgart: Ulmer.

24. Jones AE, Stolinski M, Smith RD et al. (1999) Effect of fatty acid chain length and saturation on the gastrointestinal handling and metabolic disposal of dietary fatty acids in women. Br J Nutr 81, 37-43.
25. Baer DJ, Judd JT, Kris-Etherton PM et al. (2003) Stearic acid absorption and its metabolizable energy value are minimally lower than those of other fatty acids in healthy men fed mixed diets. J Nutr 133, 4129-4134.

26. French SJ, Conlon CA, Mutuma ST et al. (2000) The effects of intestinal infusion of long-chain fatty acids on food intake in humans. Gastroenterology 119, 943-948.

27. Flint A, Helt B, Raben A et al. (2003) Effects of different dietary fat types on postprandial appetite and energy expenditure. Obes Res 11, 1449-1455.

28. Bes-Rastrollo M, Sanchez-Villegas A, de la Fuente C et al. (2006) Olive oil consumption and weight change: the SUN prospective cohort study. Lipids 41, 249-256.

29. Schulz M, Kroke A, Liese AD et al. (2002) Food groups as predictors for short-term weight changes in men and women of the EPIC-Potsdam cohort. J Nutr 132, 1335-1340.

30. Astrup A (2001) Healthy lifestyles in Europe: prevention of obesity and type II diabetes by diet and physical activity. Public Health Nutr 4, 499-515.

31. Deutsche Gesellschaft fuer Ernaehrung, Oesterreichische Gesellschaft fuer Ernaehrung, Schweizerische Gesellschaft fuer Ernaehrung, Schweizerische Vereinigung fuer Ernaehrung (2001) Referenzwerte fuer die Naebrstoffzufubr. Frankfurt am Main: Umschau/Braus.

32. Broadfield E, McKeever T, Fogarty A et al. (2003) Measuring dietary fatty acid intake: validation of a food-frequency questionnaire against $7 \mathrm{~d}$ weighed records. BrJ Nutr $\mathbf{9 0}$, 215-220.

33. Kobayashi M, Sasaki S, Kawabata T et al. (2003) Validity of a self-administered food frequency questionnaire used in the 5-year follow-up survey of the JPHC Study Cohort I to assess fatty acid intake: comparison with dietary records and serum phospholipid level. J Epidemiol 13, S64-S81.

34. Hunter DJ, Rimm EB, Sacks FM et al. (1992) Comparison of measures of fatty acid intake by subcutaneous fat aspirate, food frequency questionnaire, and diet records in a free-living population of US men. Am J Epidemiol 135, 418-427.

35. Nagel G, Zoller D, Ruf T et al. (2007) Long-term reproducibility of a food-frequency questionnaire and dietary changes in the European Prospective Investigation into Cancer and Nutrition (EPIC)-Heidelberg cohort. $\mathrm{Br} J$ Nutr 98, 194-200.

36. Niedhammer I, Bugel I, Bonenfant S et al. (2000) Validity of self-reported weight and height in the French GAZEL cohort. Int J Obes Relat Metab Disord 24, 1111-1118.

37. Voss S, Kroke A, Klipstein-Grobusch K et al. (1997) Obesity as a major determinant of underreporting in a self-administered food frequency questionnaire: results from the EPIC-Potsdam Study. Z Ernahrungswiss 36, 229-236.

38. Cust AE, Smith BJ, Chau J et al. (2008) Validity and repeatability of the EPIC physical activity questionnaire: a validation study using accelerometers as an objective measure. Int J Behav Nutr Phys Act 5, 33. 